\title{
Etude de la coagulation du lait par la présure et de la synérèse du coagulum par la méthode thrombélastographique
}

\author{
par \\ B. TARODO de la FUENTE, C. ALAIS \\ et \\ R. FRENTZ \\ Ecole Supérieure de Laiterie, Faculté des Sciences (54) Nancy
}

\section{I. - INTRODUCTION}

L'étude physique de la coagulation du lait par la présure a été abordée par des méthodes variées. Les plus simples et les plus conventionnelles consistent à mesurer uniquement le temps de floculation, par l'observation visuelle d'un film de lait s'écoulant sur les parois d'un récipient en verre (1), (2), (3), ou le temps de coagulation par l'arrêt de l'écoulement du lait emprésuré dans un tube capillaire (4), (5), (6). Des tentatives ont été faites pour rendre plus rapide et automatique la mesure du temps de coagulation, notamment celles de De Man et Batra (7) qui ont utilisé un appareil employé en hématologie ( "Clot Timer ", Mechrolab).

Des méthodes faisant appel à des instruments plus compliqués permettent de suivre en continu une partie du phénomène de la coagulation. En dehors de la spectrophotométrie utilisée par Claesson et Nitschmann (8), les méthodes les plus intéressantes, en considérant les aspects pratiques du problème, sont basées sur les variations de viscosité et d'autres propriété rhéologiques.

Ainsi, dès 1935, Christen et Virasoro (9), (10), se basant sur la constatation que l'apparition des flocons correspondait à une brusque augmentation de viscosité, ont apprécié le temps de floculation de laits emprésurés par le viscosimètre électromagnétique de Berraz. En 1963, Burnett et Scott Blair ont décrit un " torsiomètre " permettant de suivre la coagulation du lait par la présure (11), appareil qui a été perfectionné depuis (12).

Plus tard, Jacquet et Marcais (13), (14) ont utilisé le " thrombélastographe " pour mesurer les temps de coagulation et l'évolution des propriétés mécaniques des caillés ; il s'agissait de l'appareil original, d'après Hartert, conçu pour l'étude de la coagulation du 
sang. Par la suite, en collaboration avec Frentz (4), nous avons utilisé cet appareil, sous sa forme modifiée d'après les indications de Frentz (15) afin de le rendre apte à l'étude de caillés de type adynamique, comme celui que forme le lait. L'appareil ainsi modifié a été récemment dénommé « Lacto dynamographe " ; il a été utilisé également par Thomasow (16).

Dans la communication précédente (4) nous avons montré que le thrombélastographe Hellige modifié permettait d'apprécier le temps de coagulation du lait et de mesurer les propriétés mécaniques du coagulum formé dans le lait sous l'action de la présure. En ce qui concerne le premier point, des expériences préliminaires ont fait apparaître que le temps de coagulation mesuré sur les diagrammes était supérieur au temps de floculation obtenu par les techniques conventionnelles. Ceci nous a incité à faire de nouvelles expériences afin de préciser l'écart observé par rapport à la technique de Sommer et Matsen (1) prise comme technique de référence.

En ce qui concerne le deuxième point nous avons mis en évidence l'influence de la température, de la teneur en calcium et de l'extrait sec sur les différents paramètres des diagrammes thrombélastographiques qui caractérisent le début de la réaction. Nous avons poursuivi nos recherches en nous intéressant plus particulièrement à l'évolution des propriétés de coagulum, après sa formation.

On sait que lorsqu'on provoque la coagulation du lait par la présure dans certaines conditions d'acidité et de température, l'exsudation du sérum se fait spontanément et rapidement sans qu'il soit nécessaire de découper le caillé pour en accélérer la synérèse. Le même phénomène se produit dans la cuvette du thrombélastographe et, lorsque la synérèse est assez avancée, le coagulum se détache des parois de la cuve pour se ramasser autour du cylindre central ; au fur et à mesure que la mince couche de sérum qui se trouve entre la cuvette et le caillé augmente d'épaisseur, la liaison entre la cuvette et le cylindre s'affaiblit jusqu'à devenir insuffisante pour entraîner ce dernier. Sur le papier photographique, cette phase est matérialisée par un rapprochement des deux branches qui tendent à se confondre en une droite, comme le montre la figure 1. De plus, quand on modifie le déroulement de la synérèse en agissant sur les conditions de la coagulation, l'enregistrement graphique est luimême modifié.

Dans la deuxième partie des résultats, nous exposerons les expériences ayant pour but d'étudier l'influence des variations de la concentration en présure, de l'acidité, de la teneur en calcium, de l'extrait sec du lait et de la température de coagulation sur la forme des diagrammes thrombélastographiques, en vue d'éclaircir le phénomène de la synérèse du coagulum. Nous ferons également une comparaison avec la mesure volumétrique du lactosérum séparé. 


\section{Lait}

\section{II. - MATERIEL ET METHODES}

Pour la mesure des temps de floculation, le lait de mélange utilisé provenait d'un bac de 400 litres et était écrémé le matin même du ramassage. Pendant les essais le lait, additionné de 0,5 ml

Figure 1

Diagramme thrombélastographique montrant la rétraction du tracé correspondant à la synérèse (lait reconstitué à 12 p. 100, $\left.\mathrm{pH} 5,4,35^{\circ} \mathrm{C}\right)$.

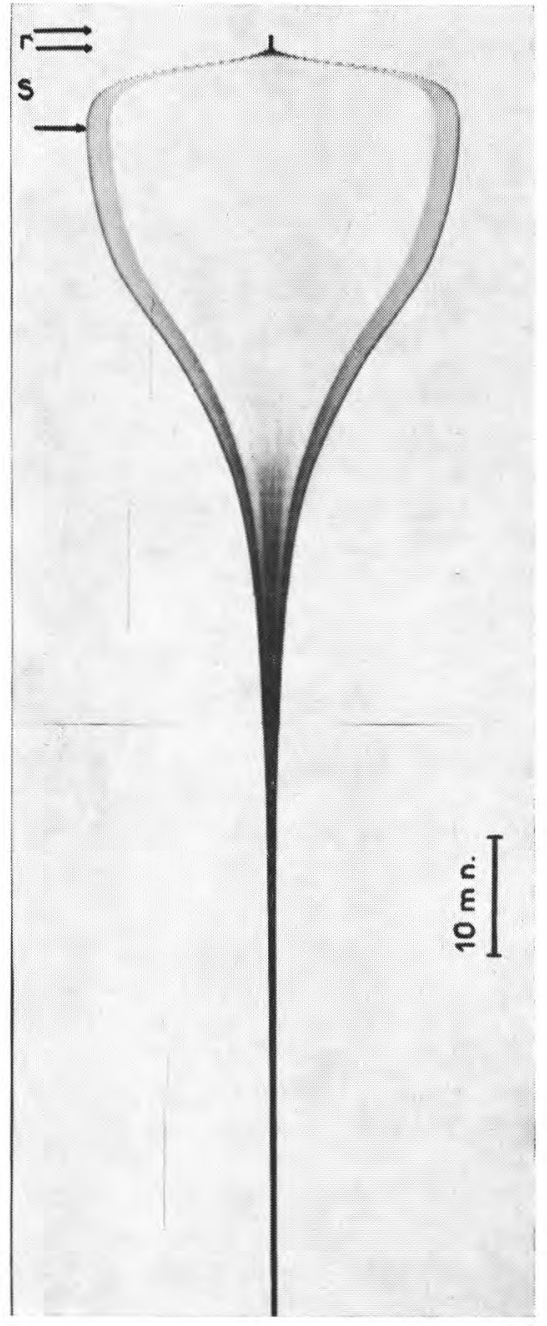


de toluène par litre, était maintenu à la température de $12^{\circ} \mathrm{C}$. Pour l'étude thrombélastographique de la synérèse, nous avons utilisé d'abord du lait en poudre instantané du commerce (lait $\mathrm{n}^{\circ} 1$ ), puis dès que nous l'avons eu à notre disposition, du lait en poudre spécial (lait $\left.n^{\circ} 2\right)^{*}$, n'ayant subi qu'un traitement thermique limité (voir réf. 17). Ce dernier lait possède une aptitude à la coagulation par la présure très supérieure à celle des laits en poudre ordinaires, comme le montre la figure 2: dans les mêmes conditions, le lait $n^{\circ} 2$ coagule plus vite que le lait $n^{\circ} 1$ et le coagulum qu'il forme acquiert beaucoup plus tôt une plus grande fermeté. Le tableau 1 montre en outre que le lait $n^{\circ} 2$ est moins modifié que le lait $n^{\circ} 1$ en ce qui concerne la sensibilité aux ions calcium.

Figure 2

Diagrammes thrombélastographiques obtenus avec les laits en poudre $\mathrm{n}^{\circ} 1$ et 2 (voir texte) (lait reconstitué à $12 \mathrm{p} .100, \mathrm{pH} 6,6$, $\left.35^{\circ} \mathrm{C}\right)$.
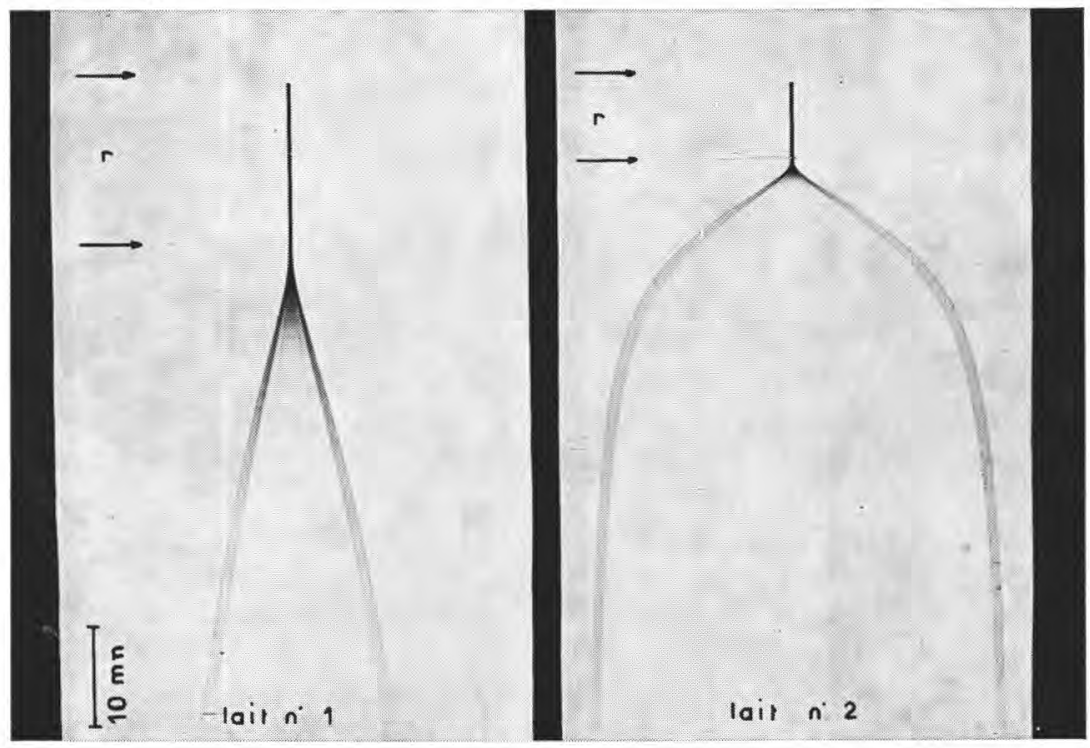

TABLEAU 1

Coagulabilité des laits en poudre

\begin{tabular}{c|c|c}
\hline $\begin{array}{c}\text { Calcium ajouté } \\
\left(\mathrm{CaCl}_{2} 10 \mathrm{p} .100\right)\end{array}$ & \multicolumn{2}{|c}{ Temps de coagulation ( $\mathrm{r}$ en minutes) } \\
\cline { 2 - 3 } 0 & Lait $\mathrm{n}^{\circ} 1$ & Lait $\mathrm{n}^{\circ} 2$ \\
\hline $0,2 \mathrm{ml} \mathrm{p.} 100 \mathrm{ml}$ & 32,5 & 11,75 \\
\hline
\end{tabular}

* Nous remercions les Etablissements Lesaffre, Cérences (50), pour la fourniture gracieuse du lait écrémé sec utilisé dans nos expériences. 
Ces laits sont reconstitués dans l'eau distillée à raison de 10 ou $12 \mathrm{~g}$ de poudre pour $100 \mathrm{ml}$ de solution, avec des doses variables de chlorure de calcium et leur $\mathrm{pH}$ est ajusté à la valeur voulue par addition d'acide lactique.

\section{Présure}

On a utilisé une solution contenant $0,75 \mathrm{~g}$ de présure en poudre du commerce au $1 / 100000$, dans $100 \mathrm{ml}$ de tampon phosphate $0,025 \mathrm{M}$ à $\mathrm{pH} 6,63$, après filtration sur verre fritté.

\section{Mesure du temps de floculation}

Le lait emprésuré est versé dans un tube en verre pyrex de $32 \mathrm{~mm}$ de diamètre intérieur tournant lentement autour de son axe incliné à $30^{\circ}$ par rapport à la surface du bain-marie. Ce tube est immergé de façon telle que le niveau du lait soit au-dessous du niveau de l'eau. L'observation du film de lait apparent sur la partie supérieure du tube permet de déterminer le temps de floculation à quelques secondes près. La mesure se fait à $35^{\circ}$ avec $50 \mathrm{ml}$ de lait de mélange cru additionné de $1 \mathrm{ml}$ de solution de présure.

\section{Technique thrombélastographique}

L'appareil utilisé est un thrombélastrographe Hellige à trois sensibilités et à température variable, précédemment décrit (15) (4). Le spot lumineux réfléchi par le miroir solidaire du cylindre plongeur est reçu sur le papier photographique qui se déroule à la vitesse de $2 \mathrm{~mm}$ par minute; il ne transmet les oscillations de la cuve que lorsque le lait commence à coaguler. Entre le prélèvement de $0,36 \mathrm{ml}$ de lait nécessaire à l'épreuve et le remplissage de la cuvette, il s'écoule environ $7 \mathrm{~s}$, temps suffisant pour provoquer un léger refroidissement du lait, ce qui est une imperfection de la technique.

Le temps de coagulation « $r$ » (fig. 1 et 2 ) correspond au temps écoulé entre l'emprésurage et le dédoublement du tracé et non plus, comme on le faisait auparavant, au temps écoulé entre l'emprésurage et le moment où l'écartement du tracé atteignait $1 \mathrm{~mm}$. Cette façon d'opérer permet une mesure plus facile et elle a l'avantage de diminuer ce paramètre en le rapprochant du temps de floculation obtenu par la technique précédemment décrite.

Le paramètre « $S$ » (fig. 1) représente le temps compris entre le début de la coagulation et le moment où le tracé thrombélastographique a atteint son amplitude maximale "Am» et commence à se rétracter.

\section{Appréciation volumétrique de la synérèse}

Nous avons imaginé plusieurs dispositifs permettant de suivre la synérèse par d'autres moyens que la méthode thrombélastographique ; ils seront décrits en détails dans une prochaine publica- 
tion. Nous avons utilisé ici, dans un but de comparaison, un des plus simples : le lait est emprésuré dans un tube en cuivre thermostaté (ce matériau évite l'adhérence du coagulum aux parois, contrairement au verre et à l'acier inoxydable). Le tube est percé d'un trou à sa partie inférieure ; il est muni d'un tamis, formant bouchon, qui permet de soulever le caillé au temps « $\mathrm{S}$ » sans le briser ; dès ce moment, le sérum s'écoule librement dans un collecteur de fraction.

On peut ainsi tracer la courbe traduisant la vitesse d'exsudation du sérum.

\section{III. - RESULTATS}

\section{a) Temps de floculation et temps de coagulation thrombélasto- graphique}

Nous avons effectué cinq séries d'essais portant sur des laits de mélange crus et écrémés, en comparant chaque fois le temps de floculation visible au temps de coagulation thrombélastographique. Toutes les séries ont donné des résultats semblables; le tableau 2 montre les temps obtenus pour l'une d'elles.

La technique thrombélastographique donne des résultats plus variables que ceux de la méthode conventionnelle ; de plus elle donne des temps sensiblement plus longs.

Il est possible que l'écart observé entre les deux méthodes varie avec la concentration en présure. En effet, le cylindre plongeur doit être entraîné d'autant plus tôt que les propriétés mécaniques du caillé évoluent plus vite, donc que le "caractère présure » est plus marqué.

\section{b) Relation entre la synérèse du coagulum et la rétraction du tracé thrombélastographique}

\section{1) Influence de l'acidité}

On a utilisé la solution de lait en poudre $\mathrm{n}^{\circ} 1$ à 12 p. 100 additionnée d'acide lactique pour amener son $\mathrm{pH}$ à la valeur désirée et l'on ajoute du chlorure de calcium à la concentration de 2,7 mM. L'emprésurage est effectué à $35^{\circ} \mathrm{C}$ par $2 \mathrm{ml}$ de solution coagulante pour $100 \mathrm{ml}$ de lait.

A pH 6,55 le temps de coagulation est de $15 \mathrm{mn}$ et il n'y a pas de rétraction du tracé au bout de $10 \mathrm{~h}$. De plus, on ne constate pas d'exsudation de sérum après $24 \mathrm{~h}$ dans le tube où a été fait l'emprésurage. A pH 6,34 et 6,10 il n'y a également pas de rétraction du tracé au bout de $10 \mathrm{~h}$ mais on peut observer une exsudation de sérum après $24 \mathrm{~h}$; par contre, à $\mathrm{pH} 5,91$ on observe un début de

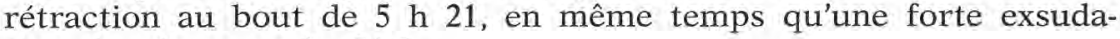
tion de sérum après $24 \mathrm{~h}$. 
La figure 3 montre les variations du temps au bout duquel la rétraction commence à se produire (paramètre $\mathrm{S}$ ) et de l'amplitude maximale "Am " en fonction du $\mathrm{pH}$, dans la zone où il est possible de faire des mesures, de 5,3 à 5,9. La rétraction a lieu d'autant plus tôt que le $\mathrm{pH}$ est plus bas ; pour le dernier essai à $\mathrm{pH} 5,35$ elle commence $9 \mathrm{mn} 30 \mathrm{~s}$ seulement après l'emprésurage.

TABLEAU 2

Temps de coagulation du lait cru $(\mathrm{pH} 6,6)$

\begin{tabular}{c|c|c}
\hline \multirow{2}{*}{$\mathrm{N}^{\circ}$} & \multicolumn{2}{|c}{ Temps en secondes } \\
\cline { 2 - 3 } & Floculation visible & Paramètre " $\mathrm{r}$ " \\
\hline & & 270 \\
2 & 267 & 285 \\
3 & 257 & 270 \\
4 & 262 & 276 \\
5 & 264 & 279 \\
6 & 264 & 273 \\
7 & 265 & 270 \\
8 & 264 & 285 \\
9 & 266 & 279 \\
10 & 264 & 288 \\
& 271 & 277,5 \\
Moyenne & 264,4 & 6,8 \\
& & \\
Ecart & 3,6 & \\
type & & \\
\hline
\end{tabular}

Figure 3

Influence du $\mathrm{pH}$ du lait sur les paramètres " $\mathrm{S}$ " et " $\mathrm{Am}$ " (voir texte)

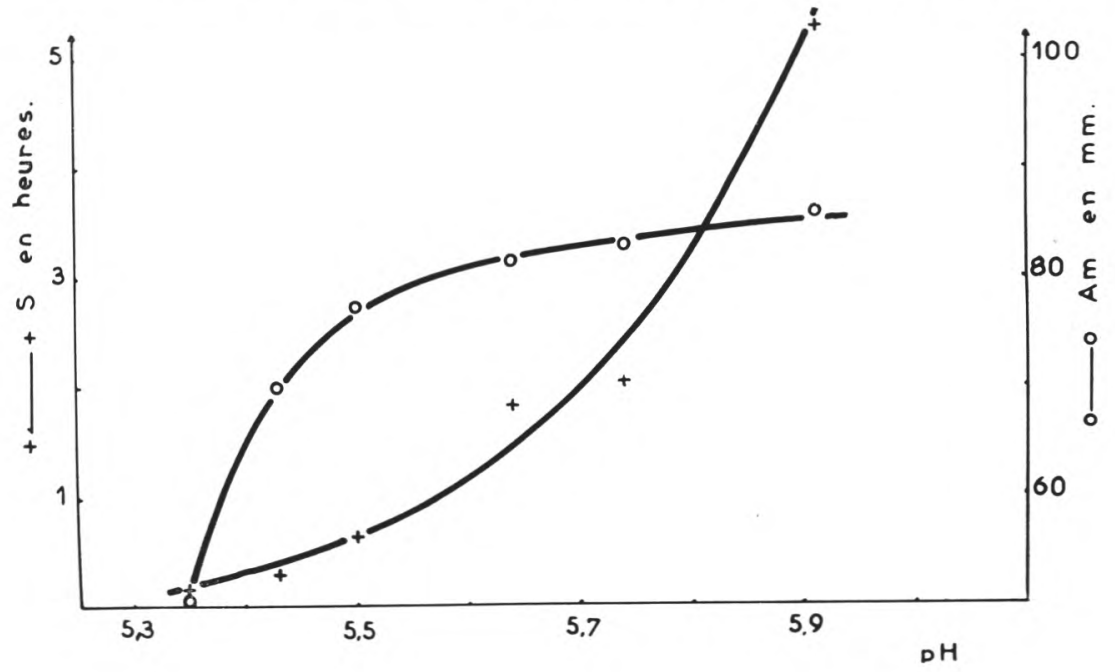


Les propriétés mécaniques du caillé, représentées par «Am ", diminuent également avec le $\mathrm{pH}$, mais pas de la même manière que le paramètre « $S$ ». La diminution est d'abord lente, puis devient rapide à partir de $\mathrm{pH} \mathrm{5,6.}$

2) Influence de l'extrait sec

Le lait a été reconstitué à partir de la poudre $n^{\circ} 1$ à des concentrations variant de 7 à 14 p. 100. La quantité de chlorure de calcium ajoutée est proportionnelle à l'extrait sec, soit une concentration de $2,7 \mathrm{mM}$ dans la solution de lait reconstitué à 10 p. 100. Les essais sont tous effectués à $\mathrm{pH} 5,5$ et à $35^{\circ} \mathrm{C}$ en gardant constante la quantité de présure utilisée : $1 \mathrm{ml}$ de solution coagulante pour $100 \mathrm{ml}$ de lait.

La figure 4 montre que le temps « $S$ » nécessaire pour obtenir le début de la rétraction augmente avec l'extrait sec du lait. Elle indique également que l'amplitude maximale "Am » évolue dans le même sens que " $S$ » mais d'une manière différente ; elle augmente rapidement pour des taux de 7 à 10 p. 100 , puis plus lentement au-dessus de cette dernière concentration, qui, il faut le remarquer, correspond à celle du lait. Il existe une similitude entre les courbes de la figure 3 et celles de la figure 4 ; l'augmentation du $\mathrm{pH}$ et celle de l'extrait sec produisent des effets comparables.

\section{Figure 4}

Influence de l'extrait sec du lait sur les paramètres " $\mathrm{S}$ " et " Am " (voir texte)

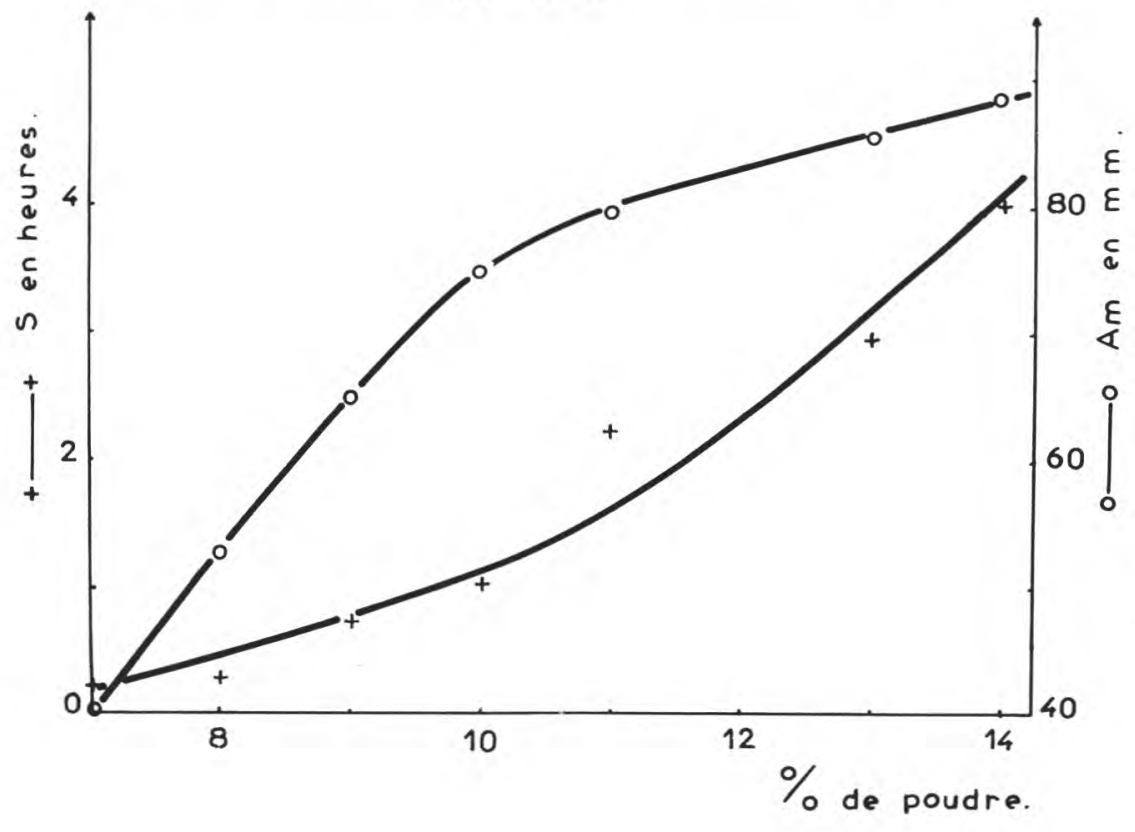




\section{3) Influence de la teneur en calcium}

On a encore utilisé le lait $n^{\circ} 1$ reconstitué à la concentration de 12 p. 100 , mais on a fait varier la quantité de calcium ajouté de 0,45 à $6,23 \mathrm{mM}$. Le $\mathrm{pH}$ et la dose de présure sont les mêmes que dans l'essai précédent.

La figure 5 montre qu'à $\mathrm{pH} 5,5$ la rétraction est d'abord nettement retardée par l'augmentation de la teneur en calcium, puis " $\mathrm{S}$ " semble se stabiliser à partir de $3,5 \mathrm{mM}$. Les propriétés mécaniques du coagulum "Am» évoluent dans le même sens, mais les variations sont minimes, de 79 à $83 \mathrm{mM}$. L'influence du calcium sur la fermeté du caillé est donc très faible à cette valeur du $\mathrm{pH}$.

Figure 5

Influence de la teneur en calcium sur les paramètres « $\mathrm{S}$ » et «Am » (voir texte)

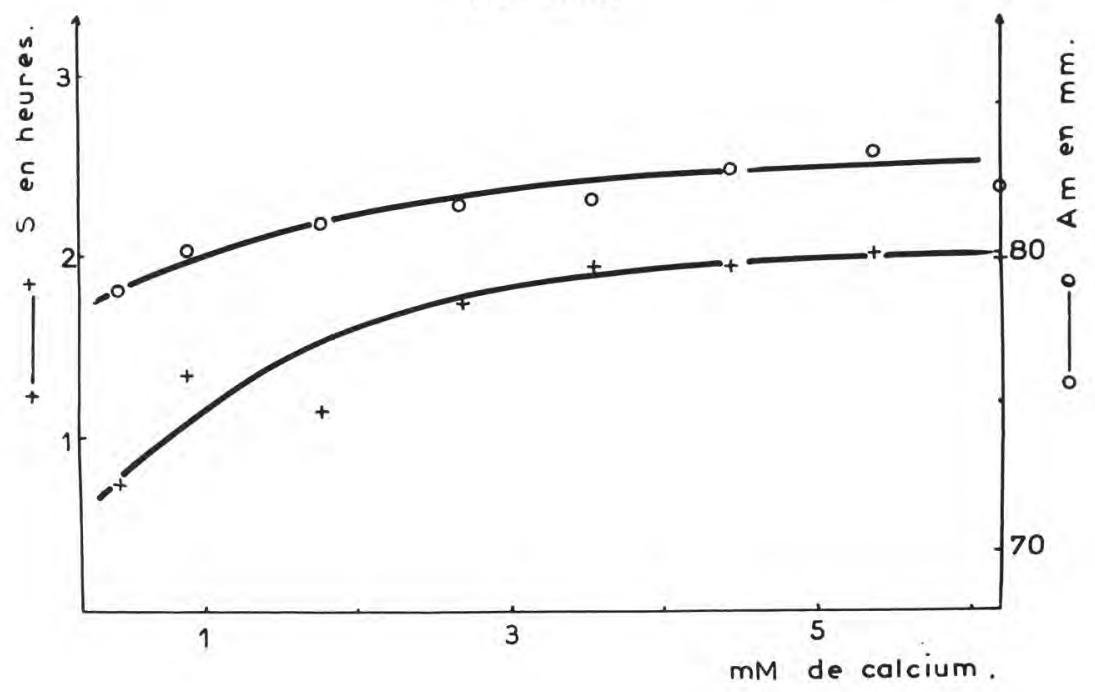

4) Influence de la température

On a utilisé cette fois-ci le lait $\mathrm{n}^{\circ} 2$ reconstitué à $12 \mathrm{p} .100$, la concentration en calcium ajouté étant 1,8 $\mathrm{mM}$, les essais sont faits à $\mathrm{pH} 5,4$. La concentration en présure reste la même que dans les essais précédents mais on fait varier la température de 24 à $48^{\circ} \mathrm{C}$. La figure 6 montre que l'influence de la température sur la rétraction du tracé est très sensible. A $24^{\circ} \mathrm{C}$ le début de la rétraction intervient $110 \mathrm{mn}$ après la floculation ; à des températures voisines de $40^{\circ} \mathrm{C}$, ce temps n'est plus que de quelques minutes. Les propriétés mécaniques du coagulum, mesurées par «Am», diminuent à mesure que la température augmente. L'augmentation de la tempé- 
rature et la diminution du $\mathrm{pH}$ ont des effets comparables (fig. 6 et 3) : elles accélèrent l'apparition du maximum des propriétés mécaniques mais elles provoquent en même temps une diminution rapide de ces dernières.

5) Influence de la concentration en présure

Dans cette expérience on a utilisé les deux laits $\mathrm{n}^{\circ} 1$ et $\mathrm{n}^{\circ} 2$ reconstitués à 10 p. 100 , à la température de $35^{\circ} \mathrm{C}$. La solution du lait $\mathrm{n}^{\circ} 1$ était à $\mathrm{pH} 5,7$, avec $2,7 \mathrm{mM}$ de calcium ajouté, et celle du lait $\mathrm{n}^{\circ} 2$ à $\mathrm{pH} 6,7$, avec $1,8 \mathrm{mM}$ de calcium ajouté.

Les deux essais ont donné des résultats comparables, bien qu'il s'agisse de laits différents par leur aptitude à la coagulation et utilisés à des valeurs de $\mathrm{pH}$ assez éloignées l'une de l'autre.

Figure 6

Influence de la température sur les paramètres « $\mathrm{S}$ » et « $\mathrm{Am}$ »

(voir texte)

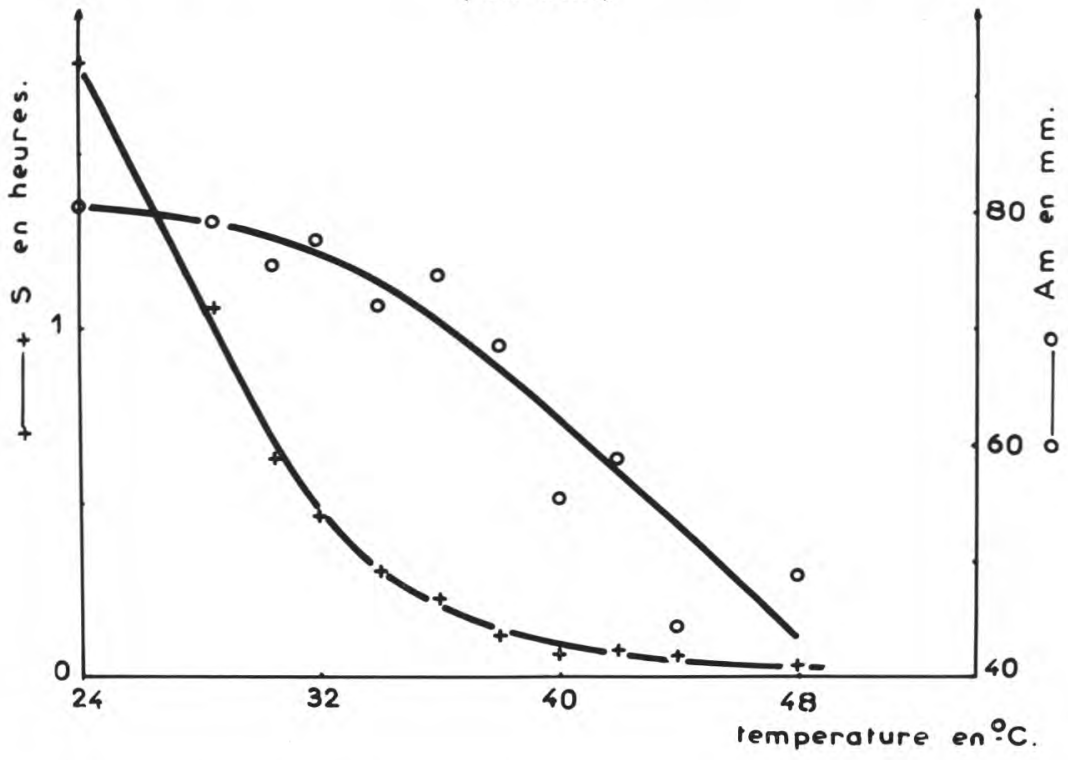

La rétraction est d'abord favorisée par l'augmentation de la dose de présure, « $S$ » diminue assez rapidement, puis, à partir d'une certaine concentration en enzyme, la valeur de ce paramètre ne change plus. Par contre, il est évident que les propriétés mécaniques du coagulum, représentées par "Am », ne sont pas influencées par la dose de présure, dans l'intervalle considéré. La figure 7 schématise les résultats obtenus avec le lait no 2 à $\mathrm{pH} \mathrm{6,7;} \mathrm{la} \mathrm{dose}$ de présure variant de 1 à 5 . 
La figure 7, montre encore que le lait no 2 permet des observations sur le déroulement de la rétraction à des valeurs de $\mathrm{pH}$ relativement élevée $(6,7)$; nous avons vu précédemment qu'avec le lait $\mathrm{n}^{\circ} 1$ les observations ne sont possibles qu'en dessous de $\mathrm{pH} 6,0$.

Figure 7

Influence de la concentration en présure sur les paramètres " $\mathrm{S}$ " et " $\mathrm{Am}$ "

(voir texte)

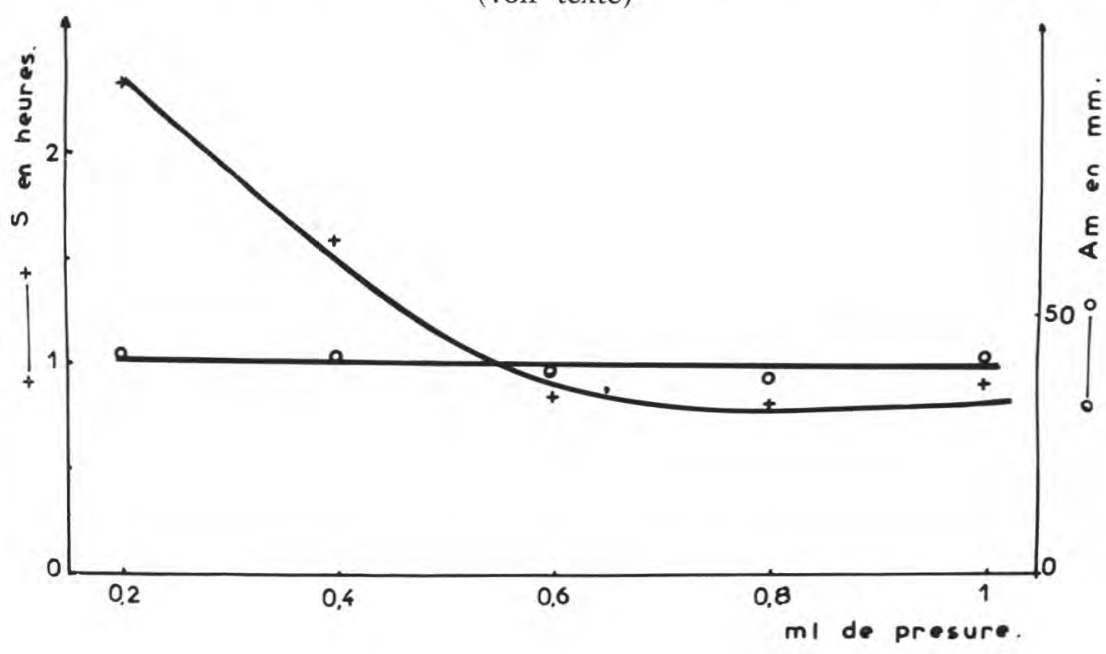

6) Courbes obtenues par la méthode thrombélastographique et la méthode d'appréciation volumétrique du sérum.

Les essais ont été effectués avec le lait no 2 reconstitué à 12 p. 100

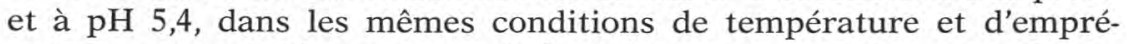
surage que précédemment et à la même concentration en calcium ajouté : 1,8 mM. Les courbes de la figure 8 représentent, en fonction du temps, d'une part, la quantité totale de sérum recueilli et, d'autre part, la rétraction du tracé thrombélastographique (en ordonnée : différence entre «Am » et l'amplitude au temps considéré) en prenant le point « $\mathrm{S}$ » comme origine des temps.

On observe une évolution semblable du volume de sérum recueilli et de la rétraction du tracé. Cependant les courbes ne sont pas superposables : dans les deux cas, elles changent nettement de pente, mais après des temps différents ; celle du sérum écoulé vers 8-10 mn, celle du tracé vers $25 \mathrm{mn}$. D'autre part, ces deux courbes tendent vers un palier, celle du sérum un peu plus rapidement que celle du tracé. Dans la discussion nous reviendrons sur l'interprétation de ces résultats. 
Figure 8

Courbes représentant la synérèse du coagulum :

0 - 0 exsudation du sérum

$\mathrm{x}-\mathrm{X}$ rétraction $\mathrm{du}$ tracé thrombélastographique

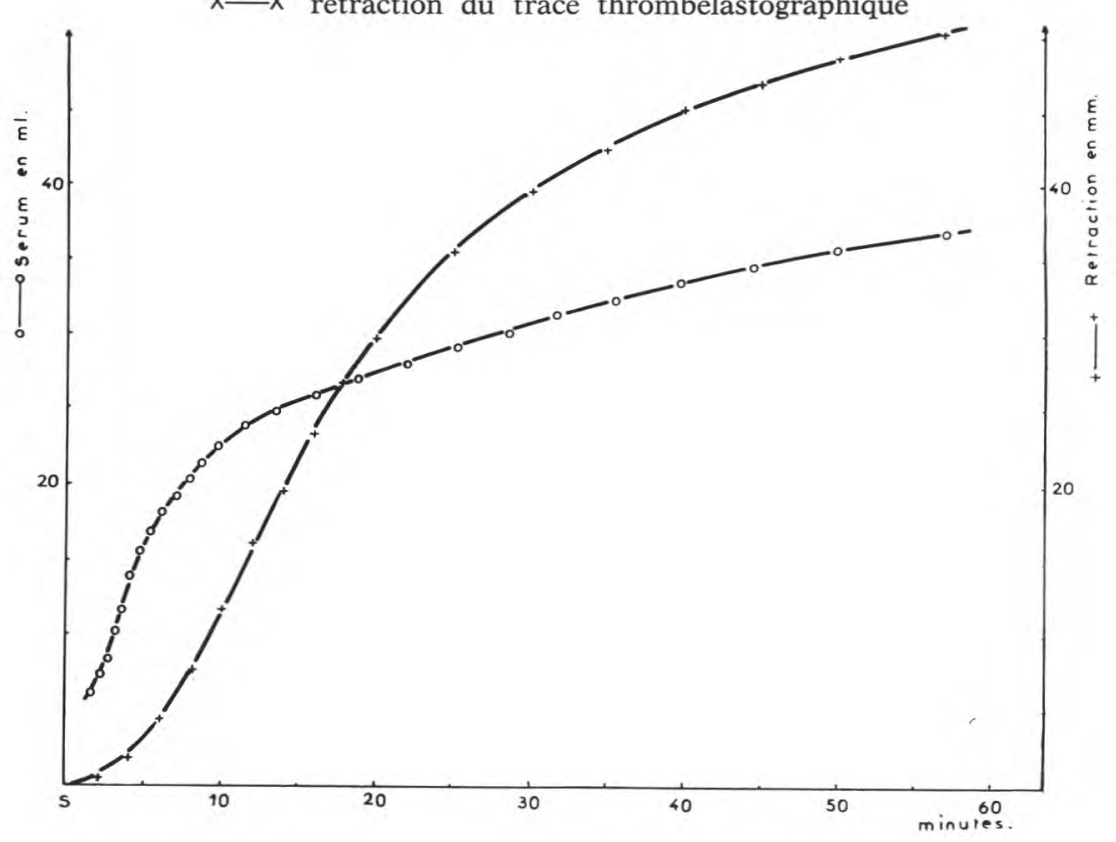

\section{IV. - DISCUSSION}

Le temps de coagulation du lait est obtenu avec un peu plus de précision par la méthode conventionnelle d'observation de la floculation que par la méthode thrombélastographique. Le paramètre " $\mathbf{r}$ » est toujours plus grand que le temps de floculation visible (4) (16). Cet allongement ne peut s'expliquer complètement par le fait que le lait subit, au cours du prélèvement, une légère baisse de température ; la plus grande partie de l'écart constaté est inhérente au principe même de la technique. En effet, le temps de coagulation est ici déterminé par la variation de consistance du milieu, puisque le cylindre plongeur n'est entraîné que lorsque le gel en formation a atteint une consistance suffisante pour vaincre la résistance du fil de torsion.

En ce qui concerne la formation et l'évolution du coagulum, par contre, le tracé thrombélastographique a permis d'intéressantes observations. La comparaison de nos résultats avec les travaux effectués par de nombreux auteurs utilisant d'autres méthodes (18) à (26), 
permet de constater que la rétraction du tracé varie de la même manière que la synérèse d'un " caillé présure " quand on modifie les conditions de la coagulation. Or, l'exsudation du sérum, c'est-àdire l'égouttage du caillé, est un phénomène très important en fromagerie, dont le déroulement dépend beaucoup des changements de température, de l'acidification et des modifications de l'équilibre physico-chimique, et aussi des actions mécaniques.

L'étude des principaux facteurs, excepté la concentration en présure, montre une similitude dans leurs effets. Une augmentation des propriétés mécaniques représentées par «Am» et un allongement concomittant du temps « $S$ » au bout duquel commence la rétraction, s'observent lorsque :

- le $\mathrm{pH}$ augmente,

- l'extrait sec augmente,

- le calcium ajouté augmente,

- la température diminue.

Dans la pratique, on considère plutôt l'abaissement du $\mathrm{pH}$ du lait que son augmentation ; comme il entraîne une solubilisation progressive des cations fixés par la caséine, et en particulier du calcium, il en découle une diminution des propriétés mécaniques du coagulum ; ce qui est en accord avec les conclusions de Mc Gann et

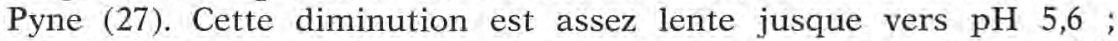
elle devient plus rapide à mesure que le "caractère lactique " s'affirme. Il est à noter d'ailleurs que ce "caractère lactique ", c'està-dire la formation d'un caillé moins élastique et moins ferme, apparaît assez brusquement dans cette zone de $\mathrm{pH}$.

L'augmentation de l'extrait sec du lait et l'addition de chlorure de calcium entraînent simultanément un accroissement de la teneur en calcium soluble et en phosphate de calcium colloïdal (28) (29) et, par conséquent, provoquent l'augmentation du paramètre "Am ». Il est à noter que, dans le premier cas, vient s'ajouter l'effet propre de la matière sèche, c'est-à-dire de la concentration en micelles de caséine par unité de volume. L'augmentation de "Am " lorsque la température diminue est, par contre, plus difficile à expliquer ; en tout cas, il est montré (fig. 6) que plus la température de coagulation est élevée, moins le caillé est ferme.

L'accélération de la rétraction par l'acidification fait intervenir au moins deux actions : celle, favorable, du $\mathrm{pH}$ sur l'activité de l'enzyme, et celle, également favorable vis-à-vis de la synérèse, de la solubilisation du calcium colloïdal. Celle-ci peut avoir pour conséquence de faciliter des rapprochements ultérieurs, par l'établissement de liaisons secondaires, comme les liaisons hydrogène, ou de ponts disulfure, entre les micelles ou les aggrégats de micelles. Au contraire, le ralentissement de la rétraction, consécutif á l'augmentation de la teneur en calcium ou de l'extrait sec peut être la consé- 
quence du masquage du fait de la salification, de groupements qui seraient actifs pendant la synérèse. L'élévation de la température diminue le paramètre « $S$ » ; c'est, comme l'abaissement du $\mathrm{pH}$, un facteur favorable à l'activité enzymatique.

La synérèse commence avec l'action de la présure sur la caséine $\chi$ par une diminution de l'eau liée entourant les micelles de caséine, ce qui est montré par la chute de viscosité observée au début de l'action de l'enzyme (30) (31). Cette enveloppe d'eau entourant les micelles et les aggrégats de micelles, est, en effet, un inhibiteur de la synérèse en ralentissant la formation des liaisons secondaires (19) (32).

La diminution du temps " $S$ " nécessaire pour obtenir le début de la rétraction, provoquée par l'accroissement de la quantité de présure, est probablement en rapport avec le phénomène de déshydratation des micelles. Tant que le rapport enzyme/substrat est inférieur au rapport optimal, l'augmentation de la quantité de présure favorise la coagulation, « $\mathrm{r} »$ et $« \mathrm{~S}$ » diminuent; au-delà de cet optimum, ces valeurs ne varient plus. On peut voir, en outre, sur la figure 7, que le paramètre «Am» ne varie pratiquement pas lorsque la concentration en présure change. Ceci semble montrer que l'augmentation de la dose de présure ne joue que sur la rapidité avec laquelle s'établissent les liaisons entre les micelles de caséine, pendant la coagulation, et non sur leur nombre maximal ou sur leur nature.

En ce qui concerne la comparaison entre les deux modes d'étude de la synérèse, nos résultats montrent que la rétraction du tracé thrombélastographique suit un cours comparable à celui de l'égouttage du coagulum. Dans les deux cas les courbes obtenues révèlent une vitesse initiale élevée du phénomène. La première partie de la courbe est plus rapide pour l'égouttage que pour la rétraction du tracé. La séparation du sérum est, en effet, fortement influencée par les actions mécaniques pouvant s'exercer sur le coagulum ; le simple soulèvement du caillé constitue une action mécanique qui entraîne une brusque expulsion du sérum et ne permet pas de connaître le début du phénomène. A cette action vient en outre s'ajouter le fait que le caillé ne baigne plus dans son sérum, ce qui, comme l'a montré Lawrence (23), favorise la synérèse. Par contre, dans le cas du thrombélastographe, le caillé subit seulement une torsion assez faible puisque l'amplitude des oscillations de la cuvette n'est que de $4^{\circ} 45^{\prime}$.

Lorsque l'égouttage se termine, la courbe représentant le volume de sérum recueilli en fonction du temps, tend vers un palier correspondant au volume maximal de sérum. Il n'en est plus ainsi dans le cas du thrombélastographe : la rétraction maximale du tracé correspond à la suppression de la liaison entre la cuvette et le cylindre et n'est pas en rapport direct avec le volume maximal de sérum qui peut être recueilli. 
La méthode thrombélastographique est très simple en ce qui concerne sa mise en œuvre, lorsque l'on dispose de l'appareil (pour une expérience, le temps de préparation est de l'ordre de $10 \mathrm{mn}$ ). L'ensemble des résultats obtenus par cette technique montre qu'elle peut donner des renseignements intéressants sur la synérèse du coagulum formé sous l'action de la présure, principalement au début du phénomène, et contribuer à son explication qui reste encore obscure. Dans ce but, nous poursuivons nos recherches, dans d'autres conditions expérimentales et en établissant des comparaisons avec d'autres méthodes d'étude de la synérèse.

\section{$R$ é $s$ u é}

La méthode thrombélastographique donne des temps de coagulation du lait légèrement plus longs que la méthode conventionnelle. Elle permet de suivre l'évolution du coagulum formé par la présure et sa synérèse. Les propriétés mécaniques du coagulum et le temps nécessaire à l'obtention de la synérèse augmentent avec le $\mathrm{pH}, \mathrm{l}$ lextrait sec et la quantité de calcium ajoutée; elles diminuent quand la température s'élève. L'accroissement de la quantité de présure n'influe pas sur les propriétés mécaniques du coagulum et n'agit sur le temps nécessaire à l'obtention de la synérèse qu'aux faibles concentrations.

\section{S u m m a ry}

The thrombelastographic method give milk-clotting times slightly longer than the conventional one. It allows us to follow the evolution of the coagulum produced by rennet and its syneresis. The firmness of the coagulum and the time for the beginning of the syneresis increase with the $\mathrm{pH}$, the total solids and the calcium concentration. On the other hand they decrease when temperature rises. The increase of the rennet concentration does not affect the rheological properties of the coagulum and act on the time required to obtain syneresis only at low concentrations.

\section{Zusammenfassung}

Die thrombelastographische Methode zeigt Milchgerinnungzeiten die etwas länger sind als bei der gebräuchlichen Methode. Damit können wir verfolgen das Werden des Labbruchs une seine Synäresis. Die mechanischen Eigenschaften des Bruchs und die notwendige Zeit für die Synäresis steigern mit dem pH-Wert, mit der trockenem Masse, mit dem Gehalt an Calciumionen und auch wenn die Temperatur abnimmt. Bei höheren Verwendungen von Lab, bleiben die mechanischen Eigenschaften des Bruchs ähnlich und die notwendige Zeit der Synäresis ist nuch bei geringer Verdichtung verändert. 


\section{Bibliographie}

[1] Sommer (H. H.), Matsen (H.). - The relation of mastitis to rennet coagulability and curd strength of milk. J. Dairy Sc., 18 (1935), p. 741-749.

[2] BerRIDGe (N. J.). - Some observations on the determination of the activity of rennet. The Analyst, 77 (1952), p. 57.

[3] BERRIDGE (N. J.). - An improved method of observing the clotting of milk containing rennin. J. Dairy Res., 19 (1952), p. 328-329.

[4] TARodo de LA Fuente (B.), Frentz (R.). - Etude thrombélastographique de la coagulation du lait par la présure : action de la température et de la concentration en calcium. Lait, 46 (1966), p. 371-393.

[5] Scott Blair (G. W.), Burnett (J.). - A simple method for detecting an early stage in coagulation of rennetted milk. J. Dairy Res., 30 (1963), p. 383.

[6] Davis (J. G.), The Marshall Bowl test. Cheese. Vol. 1 : Basic Technology. Edit. J. et A. Churchill LTD, London.

[7] De Man (J. M.), Batra (S. C). - Measurement of the rennet clotting time of milk with an automatic clot timer. Dairy Ind., 29 (1964), p. 32.

[8] Claesson (O.), Nitschmann (H.S). - Optical investigation of the rennet clotting of milk. Acta Agric. Scandinavica, VII : 4 (1957), p. 341.

[9] Christen (C.), Virasoro (E.). - La loi d'action de la présure dans la coagulation du complexe caséinate de calcium + phosphate de calcium. Lait, 12 (1932), p. 923.

[10] Christen (C.), Virasoro (E.). - Présures végétales : extraction et propriétés. Lait, 15 (1935), p. 354-496.

[11] Burnett (J.), Scott Blair (G. W.). - A speed compensated torsiometer for measuring the setting of milk by rennet. Dairy Ind., 28 (1963), p. 220.

[12] Plint (M. A.), Burnett (J.), Scott Blair (G. W.). - Note on an improved dairy torsiometer. Dairy Ind., 32 (1967), p. 104.

[13] JacQuet (J.), Marcais (H.). - Enregistrement graphique du phénomène de coagulation du lait. C.R. Ac. Agric., 50 (1964), p. $1272-1280$.

[14] Marcais (H.). - Emploi de la thrombélastographie pour l'étude de la coagulation du lait. Lait, 45 (1965), p. 241.

[15] Frentz (R.). - Application de la thrombélastographie de Hartert à l'étude de la coagulation du lait. Lait, 45 (1965), p. 489-508.

[16] THomasow (J.). - Untersuchungen an Labgallerten von milch mit dem Hellige-thrombelastographen. Milchwissenschaft, 23 (1968), p. 725.

[17] Alais (C.), NovaK (G.). - Etude d'un enzyme coagulant microbien dérivé de Endothia parasitica. I. Propriétés biochimiques de l'enzyme coagulant Pfizer et propriétés rhéologiques des caillés formés dans le lait. Lait, 48 (1968), p. 393-418.

[18] Dimov (N.D), Mineva (P.). - Some factors influencing the syneresis of fresh curd and the loss of dry matter in the whey of cow's, buffaloe's and sheep's milk. Int. Dairy Congr., B, Section IV (1962), p. 817.

[19] Cheeseman (G. C.). - Syneresis of milk and caseinate solutions. Int. Dairy Congr. B, Section IV (1962), p. 465.

[20] Schulz (M. E.), Kley (W.). - Die schrumpfung der labgallerte der milch. Milchwissenschaft, 11 (1956), p. 116.

[21] Schulz (M. E.). - Die dynamik der labgerinnung in der käserei. Milchwissenschaft, 6 (1951), p. 74-82. 
[22] Schulz (M. E.). - Die dynamik der labgerinnung in der käserei. Milchwissenschaft, 6 (1951), p. 112-118.

[23] Lawrence (A.). - Syneresis of rennet curd. I. Effect of time and temperature. II. Effect of stirring and of the volume of whey. Austr. J. Dairy Technology, 14 (1959), p. 166-172.

[24] BEEBY (R.). - A method for following the syneresis of the rennet coagulum in milk. Austr. J. Dairy Technology, 14 (1959), p. 77-79.

[25] Alars (C.). - Problèmes scientifiques posés par la transformation du lait en fromage. Ind. laitière, 224 (1965), p. 436-443.

[26] WhiteheAd (H. R.), HARKNESS (W. L.). - The influence of variations in cheesemaking procedure in the expulsion of moisture from Cheddar cheese curd. Austr. J. Dairy Technology, 9 (1954), p. 103.

[27] Mc Gann (T.C.A.), Pyne (G. T.). - The colloidal phosphate of milk. III. Nature of its association with casein. J. Dairy Res., 27 (1960), p. 403-417.

[28] VuJicic (I.), DE MAN (J. M.). - Soluble colloidal equilibria of constituents of concentrated milks. Milchwissenschaft, 21 (1966), p. 346.

[29] FRICKER (A.). - Beitrag zur frage der reifung der milch. Int. Dairy Congr. III, Section 5 (1959), p. 1276.

[30] Scott Blair (G. W.), Oosthuizen (J. C.). - A viscometric study of the breakdown of casein in milk by rennin and rennet. J. Dairy Res., 28 (1961), p. 165.

[31] Oosthuizen (J.C). - The kinetics of the rennin-casein reaction in abnormal cow's milk and milks of some other species and of the action of rennet substitutes on normal cow's milk. J. Dairy Res., 29 (1962), p. 297.

[32] KirchmeIER (O.). - Uber die enzymatische dehydratation des caseins bei labeinnirkung. Milchwissenschaft, 16 (1961), p. 137.

Reçu pour publication en mai 1969. 